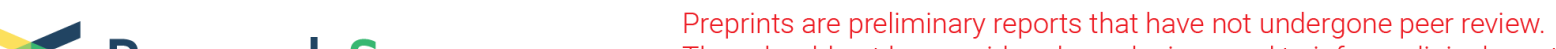

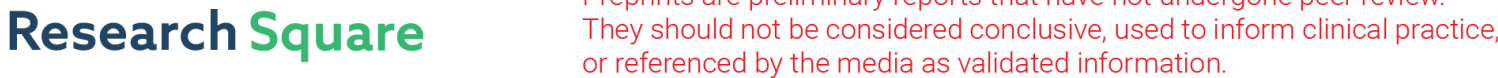

\section{Mesenchymal Stem Cells Protect Against Ferroptosis via Exosome-Mediated Stabilization of Xct in Acute Liver Injury}

\section{Feiyan Lin}

Zhejiang University First Affiliated Hospital State Key Laboratory for Diagnosis and Treatment of Infectious Diseases

\section{Wenyi Chen}

Zhejiang University First Affiliated Hospital State Key Laboratory for Diagnosis and Treatment of Infectious Diseases

\section{Jiahang Zhou}

Zhejiang University First Affiliated Hospital State Key Laboratory for Diagnosis and Treatment of Infectious Diseases

Jiaqi Zhu

Zhejiang University First Affiliated Hospital State Key Laboratory for Diagnosis and Treatment of Infectious Diseases

\section{Qigu Yao}

Zhejiang University First Affiliated Hospital State Key Laboratory for Diagnosis and Treatment of Infectious Diseases

\section{Bing Feng}

Zhejiang University First Affiliated Hospital State Key Laboratory for Diagnosis and Treatment of Infectious Diseases

\section{Xudong Feng}

Zhejiang University First Affiliated Hospital State Key Laboratory for Diagnosis and Treatment of Infectious Diseases

\section{Xiaowei Shi}

Zhejiang University First Affiliated Hospital State Key Laboratory for Diagnosis and Treatment of Infectious Diseases

\section{Qiaoling Pan}

Zhejiang University First Affiliated Hospital State Key Laboratory for Diagnosis and Treatment of Infectious Diseases

Jiong Yu

Zhejiang University First Affiliated Hospital State Key Laboratory for Diagnosis and Treatment of Infectious Diseases

\section{Lanjuan Li}


Zhejiang University First Affiliated Hospital State Key Laboratory for Diagnosis and Treatment of Infectious Diseases

Hongcui Cao ( $\nabla$ hccao@zju.edu.cn )

Zhejiang University https://orcid.org/0000-0002-6604-6867

Research

Keywords: ferroptosis, mesenchymal stem cell, lipid peroxidation

Posted Date: May 27th, 2021

DOI: https://doi.org/10.21203/rs.3.rs-530971/v1

License: (9) This work is licensed under a Creative Commons Attribution 4.0 International License. Read Full License 


\section{Abstract}

Background: Ferroptosis, a newly recognized form of regulated cell death, was recently identified as a novel therapeutic target in tissue injury. Various studies have shown that administration of mesenchymal stem cells (MSC) is a promising therapeutic approach to repair liver injury. However, the role of ferroptosis in acute liver injury (ALI) and MSC-based therapy is unknown.

Results: Here we found that $\mathrm{CCl}_{4}$ induced elevated lipid reactive oxygen species (lipid-ROS) and mRNA levels of putative molecular markers of ferroptosis such as Ptgs2 and $\mathrm{LOX}$ genes. $\mathrm{CCl}_{4}$ also downregulated the XCT protein levels resulting in the accumulation of lipid peroxidation and ferroptosis. MSC transplantation largely abolished $\mathrm{CCl}_{4}$-induced ferroptosis. Furthermore, the protective effects of MSC against ferroptosis were closely correlated with exosome-mediated stabilization of XCT. Administration of MSC-Exo restored the XCT protein level, decreased the elevated lipid-ROS level and Ptgs 2 and LOX mRNA levels, and promoted liver restoration by inhibiting ferroptosis. Interestingly, in ALI mouse livers after MSC-Exo treatment, exosome-induced recovery of XCT protein was accompanied by upregulation of CD44 and OTUB1. The level of ubiquitinated $\mathrm{xCT}$ upregulated by $\mathrm{CCl}_{4}$ was significantly downregulated by OTUB1-mediated deubiquitination, and strong interactions of XCT with OTUB1 and CD44 proteins were detected.

Conclusions: Taken together, our data indicate that MSC-Exo has a protective role against ferroptosis by maintaining $\mathrm{XCT}$ function. This provides a novel therapeutic strategy for ferroptosis-induced ALI.

\section{Background}

The liver has antibacterial/antiviral and drug detoxification functions and plays a central role in regulating homeostasis [1]. Acute liver injury (ALI) is the most prominent cause of liver disease and is associated with high morbidity and mortality [2]. A variety of hepatotoxic factors, including viruses, lipid deposition, and drugs, can induce $A L I$, and $3.5 \%$ of deaths worldwide result from liver disease $[3,4]$. Therefore, it is crucial to elucidate the underlying mechanisms of ALI and to develop appropriate treatment strategies. Carbon tetrachloride $\left(\mathrm{CCl}_{4}\right)$ is a typical hepatotoxic compound, widely used to evaluate the pathological mechanism of ALI [5]. We aimed to explore the potential mechanism of ALI using a $\mathrm{CCl}_{4}$-induced mouse model and to explore possible effective treatment strategies.

Much attention has been focused on applying mesenchymal stem cells (MSC) in biomedicine due to their potential to promote liver regeneration and repair liver injury. MSC are a heterogeneous subset of stromal cells that can be easily extracted from adipose, bone marrow, and synovial tissues and that differentiate into numerous cell lineages according to specific biomedical applications. The immunological properties of MSC, including their immunoregulatory, anti-inflammatory and immunosuppressive abilities, suggest a potential role in immune tolerance [6]. Due to these properties, MSC are considered an ideal cell source for tissue regeneration applications. Previously, we showed that transplantation of $\mathrm{MSC}$ attenuated $\mathrm{CCl}_{4^{-}}$ induced ALI by modulating the hepatic immune system [7]. Initial research suggested that MSC exert their 
therapeutic effects by engrafting to the site of injury. However, further studies showed that only a small percentage of injected MSC reach their targets and that extracellular vesicles such as exosomes released by MSC have vital and beneficial effects during treatment $[8,9]$. In addition, exosomes released from MSC contain special types of RNAs, lipids, and proteins, which are required to restore physiological conditions and assist cell and tissue regeneration and proliferation [10, 11]. MSC-derived exosomes (MSC-Exo) exhibit similar functions to those of the cells from which they originate and are applied as MSC-based cell-free therapy.

In this study, $\mathrm{CCl}_{4}$ not only induced acute injury but also promoted ferroptosis in the liver. The hepatotoxic effects of $\mathrm{CCl}_{4}$ are significant and are related to its metabolic conversion in the liver to reactive intermediates, such as trichloromethyl free radicals. In addition, lipid peroxidation stimulation was observed in the liver shortly after administering $\mathrm{CCl}_{4}$ to mice in vivo [12]. A high accumulation of products from lipid peroxidation initiates ferroptosis. In addition, various studies suggest that ferroptosis might be a new type of cell death associated with liver diseases such as viral hepatitis, drug-induced liver injury, alcoholic liver disease, non-alcoholic steatohepatitis, and hemochromatosis [13-16].

Ferroptosis is a newly identified form of regulated cell death driven by perturbation of the glutathione (GSH)-dependent lipid-hydroperoxide-scavenging network $[17,18]$. It is characterized by overproduction of lipid hydroperoxides and an increased cellular labile iron pool [19]. Lipid peroxidation is regulated by system $\mathrm{XC}^{-}$, which transports glutamate out of the cell in exchange for transport of cysteine, an important precursor for GSH synthesis, into the cell [20]. Glutathione is vital for glutathione peroxidase 4 (GPX4) activity, which protects cells against ferroptosis by converting toxic lipid hydroperoxides into nontoxic lipid alcohol [21]. Thus, inhibition of GPX4 can result in the accumulation of lipid hydroperoxides that cause cell ferroptosis. On the other hand, system $\mathrm{XC}^{-}$is a cysteine-glutamate antiporter, consisting of a light-chain subunit (SLC7A11/xCT) and a heavy-chain subunit (SLC3A2). xCT exhibits transporter activity highly specific for glutamate and cysteine and thus plays an important role in providing cysteine for the biosynthesis of GSH, a vital antioxidant in cells. Emerging strategies targeting ferroptosis have been developed to treat organ and tissue injury[22-24]. As the main detoxification organ, the liver plays a central role in regulating homeostasis. Furthermore, it was shown that MSC-derived exosomes alleviate oxidative stress-induced dysfunction in mouse livers in vivo[25].

Hence, in this study, we investigated the role of ferroptosis in MSC-based treatment for ALI in vitro and in vivo. Our results showed that XCT was significantly downregulated in an ALI mouse model, and its downregulation promoted $\mathrm{CCl}_{4}$-induced hepatocyte ferroptosis. MSC transplantation and MSC-Exo treatment largely abolished ferroptosis and protected the liver from injury. Moreover, MSC-Exo-mediated recovery of XCT protein was accompanied by upregulation of CD44 and OTUB1 in ALI mouse livers. The stability of XCT was closely related to OTUB1-mediated deubiquitination. Finally, our studies indicate that exosomes from MSC suppressed hepatocytes ferroptosis to mediate liver repair in ALI mice by maintaining $\mathrm{XCT}$ function. 


\section{Results}

\section{Ferroptosis drove $\mathrm{CCl}_{4}$-induced mouse models of $\mathrm{ALI}$}

As shown in Fig. 1A, typical histopathological changes of ALI were observed microscopically in the $\mathrm{CCl}_{4}$ administered mouse livers and compared with those in the PBS and oil groups (left and middle), including diffuse hepatic necrosis. To investigate the contribution of ferroptotic cell death in $\mathrm{CCl}_{4}$-induced ALI, we performed real-time PCR analysis of putative molecular markers of ferroptosis, which showed that $\mathrm{CCl}_{4}$ treatment induced robust increases in the mRNA levels of liver prostaglandin-endoperoxide synthase 2 (Ptgs2), 15-LOX, 12-LOX, and 5-LOX (Fig. 1B). Next, we measured lipid peroxidation in living liver cells by flow cytometry using the C11-BODIPY581/591 fluorescent probe, a canonical index of ferroptosis. $\mathrm{CCl}_{4}$ significantly increased the lipid-ROS levels in the mouse livers of the $\mathrm{CCl}_{4}$-treated group compared with the PBS and oil groups (Fig. 1C). The release of oxidized lipid mediators is a reported characteristic of ferroptosis[26]. Membranes with arachidonic acid enrichment may facilitate ferroptosis by releasing arachidonic acid metabolites (hydroxyeicosatetraenoic acid) during cell death. The levels of 20-hydroxyeicosatetraenoic acid (HETE), 15(R)-HETE, 15(S)-HETE, 12-HETE, 11-HETE, 8-HETE, and 18HETE were increased in the livers of mice $48 \mathrm{~h}$ after $\mathrm{CCl} 4$ injection (Fig. 1D). These findings suggest that ferroptosis is a crucial driver of $\mathrm{CCl}_{4}$-induced liver injury and mortality in mice.

\section{MSC transplantation alleviated $\mathrm{CCl}_{4}$-induced ferroptosis in ALI}

Oxidative stress-induced excessive accumulation of lipid hydroperoxides ultimately results in ferroptosis. We showed that MSC treatment dramatically alleviated $\mathrm{CCl}_{4}$-induced ALI due to its immunomodulatory function[7]. Moreover, MSC play important roles in reducing oxidative stress[27]. Thus, we hypothesize that MSC may have a protective role against ferroptosis by promoting lipid-ROS scavenging in $\mathrm{CCl}_{4}{ }^{-}$ induced ALI. Histopathological staining indicated that hepatic necrosis was dramatically improved following administration of MSC and ferrostatin-1 compared with the $\mathrm{CCl}_{4}$ group, indicating the effect of MSC against $\mathrm{CCl}_{4}$-induced ferroptosis in $\mathrm{ALI}$ (Fig. 2A). Compared with the $\mathrm{CCl}_{4}$, we observed significant decreases in the mRNA levels of Ptgs2, 12-LOX, 5-LOX, and 15-LOX, classic biomarkers of ferroptosis, in the MSC and Fer-1 groups (Fig. 2B). MSC treatment also significantly reduced the accumulation of $\mathrm{CCl}_{4^{-}}$ induced lipid hydroperoxides in the liver (Fig. 2C). Interestingly, according to the mRNA levels of liver Ptgs2 and LOXs, and lipid peroxidation in living cells, it is plausible that MSC and ferrostatin-1 have comparable effects on protection against ferroptosis. Compared with Fer-1, MSCs had a similar effect on protection against liver damage, as evidenced by C11-BODIPY581/591 probe staining (Fig. 2C). The decreases in the Ptgs2, 12-LOX, 5-LOX, and 15-LOX mRNA levels were similar between the MSC and Fer-1 groups (Fig. 2B). It is worth noting that LOXs (and particularly 15-LOX) have been reported as essential regulators of ferroptotic cell death as they contribute to the cellular pool of lipid hydroperoxides[28, 29]. Moreover, the increased levels of 20-hydroxyeicosatetraenoic acid (HETE), 15(R)-HETE, 15(S)-HETE, 12HETE, 11-HETE, 8-HETE, and 18-HETE induced by $\mathrm{CCl}_{4}$ was abrogated by MSC and Fer-1 treatment 
(Fig. 2D). These results suggest that the potential ability of MSC to downregulate peroxidation contributes to lipid-ROS reduction and inhibits the progression of ferroptosis.

\section{The XCT protein level was downregulated in $\mathrm{CCl}_{4}$-induced ALI but upregulated following MSC treatment} invivoandin vitro

Next, we established an acute hepatocyte injury model to detect the mechanisms underlying $\mathrm{xCT}$ regulation in ALI treated with MSC in vitro. On days 1,2,3, and 7, we detected a dramatic decrease in cell viability using the Cell Counting Kit-8 when the concentration of $\mathrm{CCl}_{4}$ increased to $10 \mathrm{mM}$ (Fig. 3A). To investigate the role of the $\mathrm{xCT}$ protein in the $\mathrm{CCl}_{4}$-induced acute hepatocyte injury model, we performed WB analysis to assess the protein expression levels according to the concentration and time of $\mathrm{CCl}_{4}$ treatment. The XCT protein level was significantly downregulated at a $\mathrm{CCl}_{4}$ concentration of $10 \mathrm{mM}$ (Fig. 3B). Furthermore, without active intervention, the $\mathrm{xCT}$ protein level decreased at $24 \mathrm{~h}$ after incubation with $\mathrm{CCl}_{4}$ and remained low at 48 and $72 \mathrm{~h}$ (Fig. 3C).

Based on the above hepatocyte model, co-culturing experiments were performed to investigate the effects of MSC on damaged hepatocytes. As shown in Fig. 3D, AST and ALT levels were significantly increased in the $\mathrm{CCl}_{4}$ group but dramatically reduced after MSC co-culture. We speculate that MSC have a protective effect in ALI via the paracrine pathway. Next, to ascertain whether co-culture with MSC also rescues the $\mathrm{CCl}_{4}$-induced decrease in the $\mathrm{XCT}$ protein level in primary hepatocytes, WB analysis was performed.

Interestingly, similar to the in vivo findings, the xCT protein level was reduced in the $\mathrm{CCl}_{4}$ group compared with the PBS group but was restored by MSC co-culture (Fig. 3E). These results suggest that a paracrine factor mediates the regulation of XCT protein levels to facilitate the protective effects of MSC against ferroptosis.

\section{MSC-derived exosomes inhibited ferroptosis in $\mathrm{CCl}_{4}$-induced ALlin vitro}

To further examine the role of MSC in ALI, we ascertained the levels of ROS and MDA. As shown in Fig. 4A, MSC co-culture with hepatocytes reduced the upregulation of ROS induced by $\mathrm{CCl}_{4}$. In addition, $\mathrm{CCl}_{4}$ induced a dramatic increase in MDA, a marker of ferroptosis, similar to erastin, while co-culture with MSC downregulated the increased MDA level, similar to Fer-1, in hepatocytes (Fig. 4B). These data indicate that MSC exert protective effects via paracrine mechanisms in ALI. Recent studies have shown that MSC produce exosomes, which can ameliorate tissue injury via the delivery of its DNA, miRNA, and protein contents[30-32]. Therefore, we extracted and identified exosomes from MSC conditioned medium. We used refrigerated transmission electron microscopy (Fig. 4C) and WB analysis (Supplementary Fig. S1D) to characterize vesicles recovered from the MSC-conditioned medium by differential ultracentrifugation and identified $30-100 \mathrm{~nm}$ vesicles that were morphologically similar to exosomes, which expressed the CD63 and CD81 exosomal markers. To determine whether hepatocytes can internalize MSC-Exo, we labeled exosomes with green lipophilic fluorescent dye (PKH67), and they were subsequently co-cultured with $\mathrm{CCl}_{4}$-induced injury hepatocytes. The hepatocytes exhibited high 
uptake efficiency, as indicated by the green fluorescent signal (Fig. 4D). Furthermore, after $24 \mathrm{~h}$ incubation with PKH67-labeled exosomes, more MSC-Exo aggregated on the cell membrane in $\mathrm{CCl}_{4}{ }^{-}$ induced injured hepatocytes, suggesting that the MSC-Exo exert anti-ferroptotic effects by fusing with the recipient cell membrane or interacting with proteins on the cell membrane. Next, we measured the lipid peroxidation of the primary hepatocytes with $\mathrm{CCl}_{4}$-induced injury after MSC-Exo treatment. Similar to erastin, $\mathrm{CCl}_{4}$ induced significant upregulation of lipid-ROS level, while MSC-Exo treatment decreased the upregulated level of lipid-ROS, similar to Fer-1 treatment (Fig. 4E). Moreover, $\mathrm{CCl}_{4}$ induced a dramatic increase in the MDA level, similar to erastin, while MSC-Exo reduced the increased MDA level, similar to Fer-1, in hepatocytes (Fig. 4F). This data suggested that MSC-derived exosomes inhibited ferroptosis in $\mathrm{CCl}_{4}$-induced $\mathrm{ALI}$ in vitro.

\section{MSC-derived exosomes inhibited ferroptosis in $\mathrm{CCl}_{4}$-induced $\mathrm{ALI}$ invivo}

Having shown that MSC-Exo inhibited ferroptosis in $\mathrm{CCl}_{4}$-induced liver injury in vitro, we investigated the effects of MSC-Exo in $\mathrm{CCl}_{4}$-induced ferroptosis in vivo. Interestingly, MSC-Exo also ameliorated hepatic necrosis and downregulated the increased ALT/AST levels induced by $\mathrm{CCl}_{4}$ in $\mathrm{ALI}$, which is consistent with the protective effects of MSC (Fig. 5A and B). In addition, measurement of MDA levels and real-time PCR analysis of molecular markers of ferroptosis showed that MSC-Exo treatment significantly downregulated MDA and liver Ptgs2, 15-LOX, 12-LOX, and 5-LOX mRNA levels induced by $\mathrm{CCl}_{4}$ (Fig. $5 \mathrm{C}$ and D). These findings suggest that MSC-Exo also inhibit ferroptosis in $\mathrm{CCl}_{4}$-induced liver injury in vivo.

\section{MSC-Exo protect against ferroptosis via stabilization of XCT in ALI}

We investigated the underlying mechanisms of the MSC-Exo-mediated protective effects against $\mathrm{CCl}_{4}{ }^{-}$ induced ferroptosis. Accumulating evidence indicates that the stability of $\mathrm{xCT}$, which is the functional subunit of a glutamate-cysteine transporter that resides on the cell membrane, is modulated by CD44v8-10[20, 33]. CD44 is a cell surface marker of MSCs[7] and a critical molecule inducing firm adhesion between MSC and injured liver tissues, which mediate the recruitment of $\mathrm{MSC}$ to $\mathrm{CCl}_{4}$-induced liver injury[34]. Moreover, we detected CD44 protein both in MSC and MSC-derived exosomes (Supplementary Fig. S1D). Therefore, we hypothesized that the CD44-xCT axis is functionally related to the anti-ferroptotic effects of MSC-Exo treatment. As shown in Fig. 6A, xCT protein levels in acute injured hepatocytes treated with $0,20,40,80$, and $160 \mu \mathrm{g}$ MSC-Exo were measured by WB analysis. Interestingly, the exosome-induced recovery of XCT protein was accompanied by upregulation of CD44 and OTUB1, key regulators of $\mathrm{XCT}$ function and $\mathrm{CD} 44$-mediated effects in ferroptosis, in a dose-dependent manner.

OTUB1, an ovarian tumor deubiquitinase family member, was previously reported to directly interact with and regulate $\mathrm{xCT}$ stability[35]. Furthermore, $\mathrm{CCl}_{4}$ administration increased the ubiquitination of $\mathrm{xCT}$, while MSC-Exo treatment downregulated the ubiquitination of XCT (Fig. 6B). These data suggest that $\mathrm{CCl}_{4^{-}}$ induced ubiquitination abolished the stabilization of $x C T$, leading to a downregulated level of $x C T$ in $A L I$. However, MSC-Exo treatment promotes the stabilization of $\mathrm{xCT}$ by reducing its ubiquitination, resulting in upregulation of XCT in ALI. 
To determine the mechanisms by which MSC-Exo exerts protective effects against ferroptosis, we performed immunofluorescence and co-immunoprecipitation analyses. Immunofluorescence analysis suggested that CD44 and xCT were co-expressed and increased in ALI after MSC-Exo treatment compared with the $\mathrm{CCl}_{4}$ and PBS groups (Fig. 6C). Next, to measure the interaction under physiological conditions, we performed co-immunoprecipitation analysis targeting endogenous proteins expressed in liver tissues of ALI after MSC-Exo treatment. As shown in Fig. 6D, the endogenous CD44 and OTUB1 proteins were co-precipitated by a XCT-specific antibody, while endogenous XCT was co-precipitated by a CD44-specific and OTUB1-specific antibody, respectively (Fig. 6E and F). Thus, we further speculated that MSC-Exo treatment promotes increases in CD44 and OTUB1 proteins and stabilizes XCT by directly interacting with and reducing its ubiquitination in $\mathrm{CCl}_{4}$-induced $\mathrm{ALI}$.

\section{MSC-Exo in the circulation localized more readily in the liver in $\mathrm{CCl}_{4}$-induced $\mathrm{ALI}$}

Next, we hypothesized that exosomes participate in tissue crosstalk during MSC-Exo treatment, resulting in exosome uptake in the recipient tissues. To determine the biodistribution of MSC-Exo after entering the circulation during treatment, we isolated MSC-Exo from MSC-conditioned medium and labeled them with lipophilic carbocyanine DiR. Next, the labeled MSC-Exo were intravenously administered via the tail to ALI mice, which were subjected to whole-body intravital imaging. In vivo fluorescent imaging showed that DiR-labeled MSC-Exo targeted the injured and normal livers at $6 \mathrm{~h}$ post-injection. There was also more fluorescence in ALI mice compared with the PBS and oil groups (Fig. 7A), indicating that MSC-Exo in circulation were more likely to aggregate in damaged livers. We performed fluorescent imaging of the lung, heart, kidney, liver, and spleen of ALI mice treated with DiR-labeled MSC-Exo to confirm this. As shown in Fig. 7B, the experiments in mice receiving labeled MSC-Exo showed significantly higher fluorescent signals in the liver compared with other organs, suggesting that MSC-Exo in circulation localized more readily in the liver in ALI mice. Neutralizing antibodies against CD44 reduced MSC-Exo targeting to the diseased liver by $30.85 \%(P<0.05)$ (Fig. 7C). These results indicated that the effect of MSC-Exo on protection against ferroptosis was associated with the function of CD44.

\section{Discussion}

Ferroptosis is a newly recognized non-apoptotic form of regulated cell death characterized by overwhelming accumulation of lipid hydroperoxides, which contribute to a wide range of pathologies, including cancer, neurodegeneration, and tissue injury[17, 36, 37]. Accumulating evidence suggests that patients with liver disease are likely to suffer from ferroptosis-regulated cell death[36, 38]. Recently, it was reported that ferroptosis occurs in $\mathrm{CCl}_{4}$-induced liver fibrosis[39]. In this study, besides increased liver necrotic areas, lipid hydroperoxides were also dramatically upregulated, suggesting accumulation of lipidROS in ALI. Furthermore, we observed a significant increase in ferroptosis-relevant genes, such as Ptgs2 and LOXs. Ptgs2, also known as cyclooxygenase-2, is induced in cells undergoing ferroptosis[21]. Thus, these results suggest that ferroptosis-mediated cell death occurs in $\mathrm{CCl}_{4}$-induced acute liver injury. We also showed that MSCs protect against ferroptosis-induced cell death, since we saw similar protective 
effects of ferrostatin-1 and MSC treatment on reducing lipid-ROS accumulation and ferroptosis-relevant gene expression in the ALI mouse model. The protective effects of MSCs were significantly attenuated when MSCs were simultaneously injected with a ferroptosis inducer such as erastin. Thus, our results show evidence of the protective role of MSCs against ferroptosis, based on the lipid hydroperoxides and ferroptosis gene expression levels. This work should be followed up with further investigation of the antiferroptotic mechanisms of MSCs.

Recently, increasing evidence has shown that most of the MSC-mediated beneficial effects in the treatment of disease are attributed to the functions of exosomes released by MSCs. MSC-Exo are extracellular vesicles 30-100 nm in diameter containing MSC-derived bioactive molecules (including mRNAs, miRNAs, chemokines, cytokines, enzymes, and other functional proteins) that regulate the survival and proliferation of recipient cells. Interestingly, exosomes from human umbilical cord bloodderived MSCs protect against ALI via intercellular communication[25]. Therefore, we investigated the route and mechanisms underlying the hepatoprotective role of MSC-Exo.

As a cysteine/glutamate antiporter, $\mathrm{XCT}$ (commonly known as SLC7A11) is a key modulator of glutathione biosynthesis and antioxidant defense in homeostasis. Emerging evidence suggests that upregulation of $\mathrm{XCT}$ promotes cell growth by suppressing ferroptosis. In this study, MSC-Exo significantly reduced the elevated levels of ALT/AST and restored the reduced XCT protein level in ALI. In vitro, MSCExo downregulated the increased MDA level and lipid ROS (specific biomarkers of ferroptosis) accumulation in hepatocytes induced by $\mathrm{CCl}_{4}$. Consistent with these findings, we confirmed that MSC-Exo treatment reduces MDA levels and ferroptosis-relevant gene expression to protect the liver from injury in vivo. Thus, we hypothesize that MSC-Exo inhibits ferroptosis in $\mathrm{CCl}_{4}$-induced $\mathrm{ALI}$ in vitro and in vivo.

Additionally, the exosome-induced recovery of XCT protein was accompanied by upregulation of CD44 and OTUB1 and strong interactions of XCT with CD44 and OTUB1 in ALI following MSC-Exo treatment. These data indicate that MSC-Exo might protect against ferroptosis via the CD44-xCT axis. More interestingly, MSC-Exo in the circulation localized more readily in the damaged liver. These results have vital implications concerning how MSC-Exo-mediated anti-ferroptotic effects are regulated in ALI.

\section{Conclusions}

In summary, we showed that ferroptosis occurs in liver tissues in an ALI mouse model. In addition, xCT was significantly reduced in ALI mouse liver tissues and $\mathrm{CCl}_{4}$-induced acute injured hepatocytes. MSC transplantation and MSC-Exo administration largely abolished ferroptosis and protected the liver from injury by maintaining XCT function. Furthermore, the protective effects of MSC-Exo against ferroptosis were correlated with the CD44-xCT axis. Together, these data indicate that MSC-Exo inhibit ferroptosis primarily by regulating the stabilization of $\mathrm{xCT}$ protein in ALI and strongly suggest that the ferroptosisrelevant pathway is a promising target, leading to valuable therapeutic strategies to treat ALI (Fig. 7D).

\section{Methods}




\section{Aim, design and setting of the study}

This study aimed to investigate the role of ferroptosis in ALI and the anti-ferroptotic effects of MSC and MSC-Exo. Carbon tetrachloride (CCl4) was administered in mice to induce ALI. The occurrence of ferroptosis was investigated by measuring the levels of lipid peroxidation, ROS, MDA and molecular markers of ferroptosis. Key axis proteins related to ferroptosis in the liver were analyzed by Western blot analysis, immunohistochemical staining, immunofluorescence staining and co-immunoprecipitation.

\section{Preparation of MSCs}

MSCs were obtained from compact bones of 1-week-old C57BL/6 male mice. Cells from passages 3-5 were used in the experiments. Detailed information is shown in the Supplementary Materials.

\section{Mouse models of ALI}

ALI induction was performed in 6-8-week old age-matched C57BL/6J male mice ( $n=10-12$ per group) by intraperitoneal injection of $3 \mathrm{~mL} / \mathrm{kg} \mathrm{CCl}_{4}$ (Sigma-Aldrich Trading Co., Shanghai, China) in coconut oil ( $v / v, 50 \%)$. Control and negative control mice were injected with PBS and coconut oil, respectively. At $6 \mathrm{~h}$ after injection of $\mathrm{CCl}_{4}$, the mice were divided into four groups: $\mathrm{CCl}_{4}$ group, injected with $100 \mu \mathrm{LBS}$ (supplemented with $2 \%$ mouse serum) through the tail vein; MSC group, injected with $5 \times 10^{5} \mathrm{MSCs}$ suspended in $100 \mu \mathrm{L}$ PBS (supplemented with $2 \%$ mouse serum) through the tail vein; Fer- 1 group, intraperitoneally injected with ferrostatin-1 (a ferroptosis inhibitor, $2.5 \mu \mathrm{mol} / \mathrm{kg}$ body weight); MSC + erastin group, simultaneous intraperitoneal injection of erastin (a ferroptosis inducer, $30 \mathrm{mg} / \mathrm{kg}$ body weight) twice every other day. The mice were sacrificed $48 \mathrm{~h}$ after MSC injection, and serum and liver tissues were collected for subsequent analysis.

\section{Isolation and characterization of exosomes}

MSCs were cultured in exosome-free medium, and the exosomes were isolated and purified by ultracentrifugation. Briefly, MSC conditioned medium was collected after $48 \mathrm{~h}$ of culture and centrifuged at $2,000 \times \mathrm{g}$ for $20 \mathrm{~min}$ to remove debris and cells. The supernatant was collected and transferred to a new sterile tube and centrifuged at $10,000 \times \mathrm{g}$ for $15 \mathrm{~min}$. The supernatant was filtered using a $0.22-\mu \mathrm{m}$ pore sterile filter, followed by ultracentrifugation at $110,000 \times \mathrm{g}$ for $70 \mathrm{~min}$ at $4^{\circ} \mathrm{C}$ to obtain exosome pellets, which were resuspended in PBS and stored at $-80^{\circ} \mathrm{C}$. We used the BCA protein assay kit (Thermo Fisher Scientific, Waltham, MA, USA; UD283191) to determine the protein content of the concentrated exosomes. Exosomes were identified by Western blot (WB) analysis of the marker proteins CD63 and CD81. The morphology of the exosomes was observed using a $120 \mathrm{kV}$ refrigerated transmission electron microscope (Tecnai Spirit Bio-TWIN, FEI Company, Hillsboro, OR, USA). The MSC-Exo concentration used to treat liver injury was $8 \mathrm{mg} / \mathrm{kg}$ body weight $(n=10)$ in the animal experiments.

\section{Isolation and incubation of primary hepatocytes}

Primary hepatocytes were harvested from 6-8-week-old C57BL/6 mice according to the rapid two-step method using collagenase perfusion[40]. We cultured the primary hepatocytes in William's E Medium 
containing 10\% FBS (Gibco Biosciences, Waltham, MA, USA), 10 mg/mL streptomycin, $100 \mathrm{lU} / \mathrm{mL}$ penicillin, 1\% ITS liquid medium supplement (Sigma-Aldrich, I3146), and $40 \mathrm{ng} / \mathrm{mL}$ dexamethasone, and observed the state of the primary hepatocytes daily.

\section{Lipid peroxidation, ROS, MDA, and cell viability measurements}

Lipid peroxidation and ROS were detected by C11-BODIPY (581/591) (D3861; Invitrogen, Carlsbad, CA, USA) and $\mathrm{H}_{2}$ DCFDA (D6883; Sigma-Aldrich) staining[16]. We used a kit (S0131S; Beyotime Biotechnology, Shanghai, China) to detect the hepatic malondialdehyde (MDA) content and the Cell Counting Kit-8 viability assay (Sigma-Aldrich) to determine cell viability.

\section{Real-time PCR analysis}

Total RNA was isolated from liver tissue using TRIzol (Invitrogen) according to the manufacturer's instructions. cDNA synthesis was carried out using the PrimeScript RT kit (Takara, Beijing, China). Quantitative real-time PCR was performed on the 7500 Real-Time PCR system (Thermo Fisher Scientific) using SYBR Green Supermix (Thermo Fisher Scientific) and specific primers (Supplementary Table S1). All experiments were performed in triplicate, and melting curve analysis was performed to monitor the specificity.

\section{WB analysis}

The following antibodies were used in the WB analysis: anti-ubiquitin (ab134953; Abcam, Cambridge, UK), xCT/SLC7A11 (D2M7A; Cell Signaling, Danvers, MA, USA), anti-OTUB1 (ab175200; Abcam, Cambridge, UK), anti-CD44 (ab189524; Abcam), anti-xCT (ab175186; Abcam), anti-CD44 (ab119348; Abcam), histone H3 (D1H2) XP® rabbit mAb (44999; Cell Signaling), rabbit monoclonal [EPR5702] to CD63 (ab134045; Abcam), rabbit monoclonal [EPR4244] to CD81 (ab109201; Abcam), and goat antirabbit IgG H\&L (HRP) (ab6721; Abcam).

\section{Immunofluorescence staining and co-immunoprecipitation}

We used mouse liver slides or fragments containing primary cells. The slides were incubated with primary antibodies at $4^{\circ} \mathrm{C}$ overnight. The following day, after incubation with the corresponding secondary antibody, the nuclei were stained with DAPI before observing by confocal laser microscopy. Coimmunoprecipitation analysis was carried out using the Pierce Co-Immunoprecipitation Kit (26149; Thermo Fisher) according to the manufacturer's instructions.

\section{Exosome labeling and tracking in mice}

The details of the exosome labeling and tracking procedures are provided in the Supplementary Materials.

\section{Statistical analysis}


The results are presented as means \pm standard deviation (SD) and were analyzed by GraphPad Prism software (GraphPad Software Inc., San Diego, CA, USA). Student's t-test was performed to compare values between two groups. We performed one-way ANOVA followed by Tukey's post-hoc test to compare data obtained from multiple groups. ${ }^{*} p<0.05$ and ${ }^{* *} p<0.001$ were considered to indicate statistical significance.

The procedures for isolation and culture of mouse MSCs, visualization of lipid peroxidation, western blotting analysis, and preparation of $\mathrm{CCl}_{4}$-induced hepatocytes injury are described in the supplementary materials.

\section{Abbreviations}

ALI, acute liver injury; MSC, mesenchymal stem cell; MSC-Exo, MSC derived exosomes; xCT, cystine/glutamate antiporter SLC7A11; $\mathrm{CCl}_{4}$, carbon tetrachloride; ALT, alanine aminotransferase; AST, glutamic oxalacetic transaminase; $B S A$, bovine serum albumin; $\mathrm{H} \otimes \mathrm{E}$, hematoxylin and eosin

\section{Declarations}

\section{Acknowledgements}

We thank the State Key Laboratory for the Diagnosis and Treatment of Infectious Diseases for the use of instrumentation and expert assistance. We also thank the technical support by the Core Facilities, Zhejiang University School of Medicine. This work was supported by grants for National Natural Science Foundation of China (No. 81971756); and Stem Cell and Translational Research from National Key Research and Development Program of China (No. 2020YFA0113003).

\section{Conflicts of interest}

The authors have declared that no conflict of interest exists.

\section{Ethics approval and consent to participate}

All animal experimental procedures were conducted according to a protocol approved by the Ethics Committee of the First Affiliated Hospital of Zhejiang University.

\section{Contributions}

F.L., L.L. and H.C. contributed to the research conception and design. F.L., W.C., JH.Z., JQ.Z. and Q.Y. performed the experiments. B.F., X.F., X.S., and J.Y. performed data analysis. F.L. wrote the manuscript. Q.P. supervised the study. H.C. drafted the manuscript. All authors reviewed and approved the final version of the manuscript.

\section{References}


1. Liu Y, Shao M, Wu Y, Yan C, Jiang S, Liu J, Dai J, Yang L, Li J, Jia W, et al: Role for the endoplasmic reticulum stress sensor IRE1alpha in liver regenerative responses. J Hepatol 2015, 62:590-598.

2. Samra YA, Hamed MF, El-Sheakh AR: Hepatoprotective effect of allicin against acetaminopheninduced liver injury: Role of inflammasome pathway, apoptosis, and liver regeneration. J Biochem Mol Toxicol 2020, 34:e22470.

3. Lee CA, Sinha S, Fitzpatrick E, Dhawan A: Hepatocyte transplantation and advancements in alternative cell sources for liver-based regenerative medicine. J Mol Med (Berl) 2018, 96:469-481.

4. Asrani SK, Devarbhavi H, Eaton J, Kamath PS: Burden of liver diseases in the world. J Hepatol 2019, 70:151-171.

5. Bhakuni GS, Bedi O, Bariwal J, Deshmukh R, Kumar P: Animal models of hepatotoxicity. Inflamm Res 2016, 65:13-24.

6. Gao F, Chiu SM, Motan DA, Zhang Z, Chen L, Ji HL, Tse HF, Fu QL, Lian Q: Mesenchymal stem cells and immunomodulation: current status and future prospects. Cell Death Dis 2016, 7:e2062.

7. Liu J, Feng B, Xu Y, Zhu J, Feng X, Chen W, Sheng X, Shi X, Pan Q, Yu J, et al: Immunomodulatory effect of mesenchymal stem cells in chemical-induced liver injury: a high-dimensional analysis. Stem Cell Res Ther 2019, 10:262.

8. Gao J, Dennis JE, Muzic RF, Lundberg M, Caplan Al: The dynamic in vivo distribution of bone marrow-derived mesenchymal stem cells after infusion. Cells Tissues Organs 2001, 169:12-20.

9. Borgovan T, Crawford L, Nwizu C, Quesenberry P: Stem cells and extracellular vesicles: biological regulators of physiology and disease. Am J Physiol Cell Physiol 2019, 317:C155-C166.

10. Xie L, Chen Z, Liu M, Huang W, Zou F, Ma X, Tao J, Guo J, Xia X, Lyu F, et al: MSC-Derived Exosomes Protect Vertebral Endplate Chondrocytes against Apoptosis and Calcification via the miR-31-5p/ATF6 Axis. Mol Ther Nucleic Acids 2020, 22:601-614.

11. Wang T, Jian Z, Baskys A, Yang J, Li J, Guo H, Hei Y, Xian P, He Z, Li Z, et al: MSC-derived exosomes protect against oxidative stress-induced skin injury via adaptive regulation of the NRF2 defense system. Biomaterials 2020, 257:120264.

12. Slater TF, Cheeseman KH, Ingold KU: Carbon tetrachloride toxicity as a model for studying freeradical mediated liver injury. Philos Trans $R$ Soc Lond B Biol Sci 1985, 311:633-645.

13. Macias-Rodriguez RU, Inzaugarat ME, Ruiz-Margain A, Nelson LJ, Trautwein C, Cubero FJ: Reclassifying Hepatic Cell Death during Liver Damage: Ferroptosis-A Novel Form of Non-Apoptotic Cell Death? Int J Mol Sci 2020, 21.

14. Yamada N, Karasawa T, Kimura H, Watanabe S, Komada T, Kamata R, Sampilvanjil A, Ito J, Nakagawa K, Kuwata $\mathrm{H}$, et al: Ferroptosis driven by radical oxidation of $\mathbf{n}-6$ polyunsaturated fatty acids mediates acetaminophen-induced acute liver failure. Cell Death Dis 2020, 11:144.

15. Liu J, He H, Wang J, Guo X, Lin H, Chen H, Jiang C, Chen L, Yao P, Tang Y: Oxidative stress-dependent frataxin inhibition mediated alcoholic hepatocytotoxicity through ferroptosis. Toxicology 2020, 445:152584. 
16. Wang H, An P, Xie E, Wu Q, Fang X, Gao H, Zhang Z, Li Y, Wang X, Zhang J, et al: Characterization of ferroptosis in murine models of hemochromatosis. Hepatology 2017, 66:449-465.

17. Dixon SJ, Lemberg KM, Lamprecht MR, Skouta R, Zaitsev EM, Gleason CE, Patel DN, Bauer AJ, Cantley AM, Yang WS, et al: Ferroptosis: an iron-dependent form of nonapoptotic cell death. Cell 2012, 149:1060-1072.

18. Meng X, Deng J, Liu F, Guo T, Liu M, Dai P, Fan A, Wang Z, Zhao Y: Triggered All-Active Metal Organic Framework: Ferroptosis Machinery Contributes to the Apoptotic Photodynamic Antitumor Therapy. Nano Lett 2019, 19:7866-7876.

19. Dixon SJ, Stockwell BR: The role of iron and reactive oxygen species in cell death. Nat Chem Biol 2014, 10:9-17.

20. Ishimoto T, Nagano O, Yae T, Tamada M, Motohara T, Oshima H, Oshima M, Ikeda T, Asaba R, Yagi H, et al: CD44 variant regulates redox status in cancer cells by stabilizing the XCT subunit of system xc(-) and thereby promotes tumor growth. Cancer Cell 2011, 19:387-400.

21. Yang WS, SriRamaratnam R, Welsch ME, Shimada K, Skouta R, Viswanathan VS, Cheah JH, Clemons PA, Shamji AF, Clish CB, et al: Regulation of ferroptotic cancer cell death by GPX4. Cell 2014, 156:317-331.

22. Kenny EM, Fidan E, Yang Q, Anthonymuthu TS, New LA, Meyer EA, Wang H, Kochanek PM, Dixon CE, Kagan VE, Bayir H: Ferroptosis Contributes to Neuronal Death and Functional Outcome After Traumatic Brain Injury. Crit Care Med 2019, 47:410-418.

23. Gao M, Monian P, Quadri N, Ramasamy R, Jiang X: Glutaminolysis and Transferrin Regulate Ferroptosis. Mol Cell 2015, 59:298-308.

24. Li Y, Feng D, Wang Z, Zhao Y, Sun R, Tian D, Liu D, Zhang F, Ning S, Yao J, Tian X: Ischemia-induced ACSL4 activation contributes to ferroptosis-mediated tissue injury in intestinal ischemia/reperfusion. Cell Death Differ 2019, 26:2284-2299.

25. Yan Y, Jiang W, Tan Y, Zou S, Zhang H, Mao F, Gong A, Qian H, Xu W: hucMSC Exosome-Derived GPX1 Is Required for the Recovery of Hepatic Oxidant Injury. Mol Ther 2017, 25:465-479.

26. Friedmann Angeli JP, Schneider M, Proneth B, Tyurina YY, Tyurin VA, Hammond VJ, Herbach N, Aichler M, Walch A, Eggenhofer $E$, et al: Inactivation of the ferroptosis regulator Gpx4 triggers acute renal failure in mice. Nat Cell Biol 2014, 16:1180-1191.

27. Li X, Michaeloudes C, Zhang Y, Wiegman CH, Adcock IM, Lian Q, Mak JCW, Bhavsar PK, Chung KF: Mesenchymal stem cells alleviate oxidative stress-induced mitochondrial dysfunction in the airways. J Allergy Clin Immunol 2018, 141:1634-1645 e1635.

28. Yang WS, Kim KJ, Gaschler MM, Patel M, Shchepinov MS, Stockwell BR: Peroxidation of polyunsaturated fatty acids by lipoxygenases drives ferroptosis. Proc Natl Acad Sci U S A 2016, 113:E4966-4975.

29. Shah R, Shchepinov MS, Pratt DA: Resolving the Role of Lipoxygenases in the Initiation and Execution of Ferroptosis. ACS Cent Sci 2018, 4:387-396. 
30. Li L, Zhang Y, Mu J, Chen J, Zhang C, Cao H, Gao J: Transplantation of Human Mesenchymal StemCell-Derived Exosomes Immobilized in an Adhesive Hydrogel for Effective Treatment of Spinal Cord Injury. Nano Lett 2020, 20:4298-4305.

31. Cao J, Wang B, Tang T, Lv L, Ding Z, Li Z, Hu R, Wei Q, Shen A, Fu Y, Liu B: Three-dimensional culture of MSCs produces exosomes with improved yield and enhanced therapeutic efficacy for cisplatininduced acute kidney injury. Stem Cell Res Ther 2020, 11:206.

32. Chen Y, Li J, Ma B, Li N, Wang S, Sun Z, Xue C, Han Q, Wei J, Zhao RC: MSC-derived exosomes promote recovery from traumatic brain injury via microglia/macrophages in rat. Aging (Albany NY) 2020, 12:18274-18296.

33. Ju HQ, Lu YX, Chen DL, Tian T, Mo HY, Wei XL, Liao JW, Wang F, Zeng ZL, Pelicano H, et al: Redox Regulation of Stem-like Cells Though the CD44v-xCT Axis in Colorectal Cancer: Mechanisms and Therapeutic Implications. Theranostics 2016, 6:1160-1175.

34. Aldridge V, Garg A, Davies N, Bartlett DC, Youster J, Beard H, Kavanagh DP, Kalia N, Frampton J, Lalor PF, Newsome PN: Human mesenchymal stem cells are recruited to injured liver in a beta1-integrin and CD44 dependent manner. Hepatology 2012, 56:1063-1073.

35. Liu T, Jiang L, Tavana O, Gu W: The Deubiquitylase OTUB1 Mediates Ferroptosis via Stabilization of SLC7A11. Cancer Res 2019, 79:1913-1924.

36. Zhang Z, Yao Z, Wang L, Ding H, Shao J, Chen A, Zhang F, Zheng S: Activation of ferritinophagy is required for the RNA-binding protein ELAVL1/HuR to regulate ferroptosis in hepatic stellate cells. Autophagy 2018, 14:2083-2103.

37. Hambright WS, Fonseca RS, Chen L, Na R, Ran Q: Ablation of ferroptosis regulator glutathione peroxidase 4 in forebrain neurons promotes cognitive impairment and neurodegeneration. Redox Biol 2017, 12:8-17.

38. Zhang X, Du L, Qiao Y, Zhang X, Zheng W, Wu Q, Chen Y, Zhu G, Liu Y, Bian Z, et al: Ferroptosis is governed by differential regulation of transcription in liver cancer. Redox Biol 2019, 24:101211.

39. Wang L, Zhang Z, Li M, Wang F, Jia Y, Zhang F, Shao J, Chen A, Zheng S: P53-dependent induction of ferroptosis is required for artemether to alleviate carbon tetrachloride-induced liver fibrosis and hepatic stellate cell activation. IUBMB Life 2019, 71:45-56.

40. Severgnini M, Sherman J, Sehgal A, Jayaprakash NK, Aubin J, Wang G, Zhang L, Peng CG, Yucius K, Butler J, Fitzgerald K: A rapid two-step method for isolation of functional primary mouse hepatocytes: cell characterization and asialoglycoprotein receptor based assay development. Cytotechnology 2012, 64:187-195.

\section{Supplementary}

Figure $s 1$ is not available with this version

\section{Figures}


A

Control

Oil

$\mathrm{CCl}_{4}$
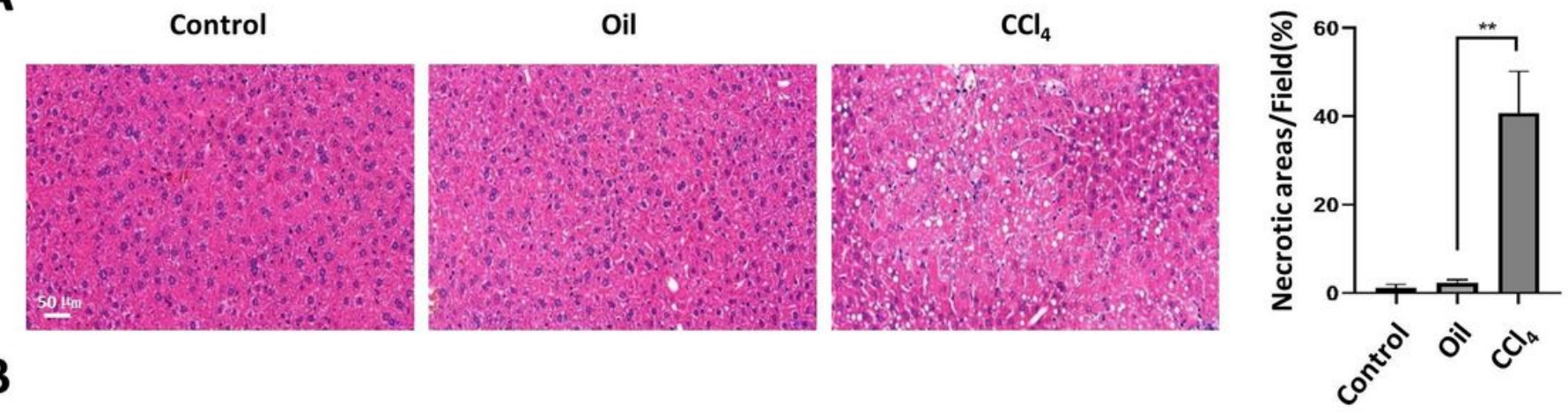

B
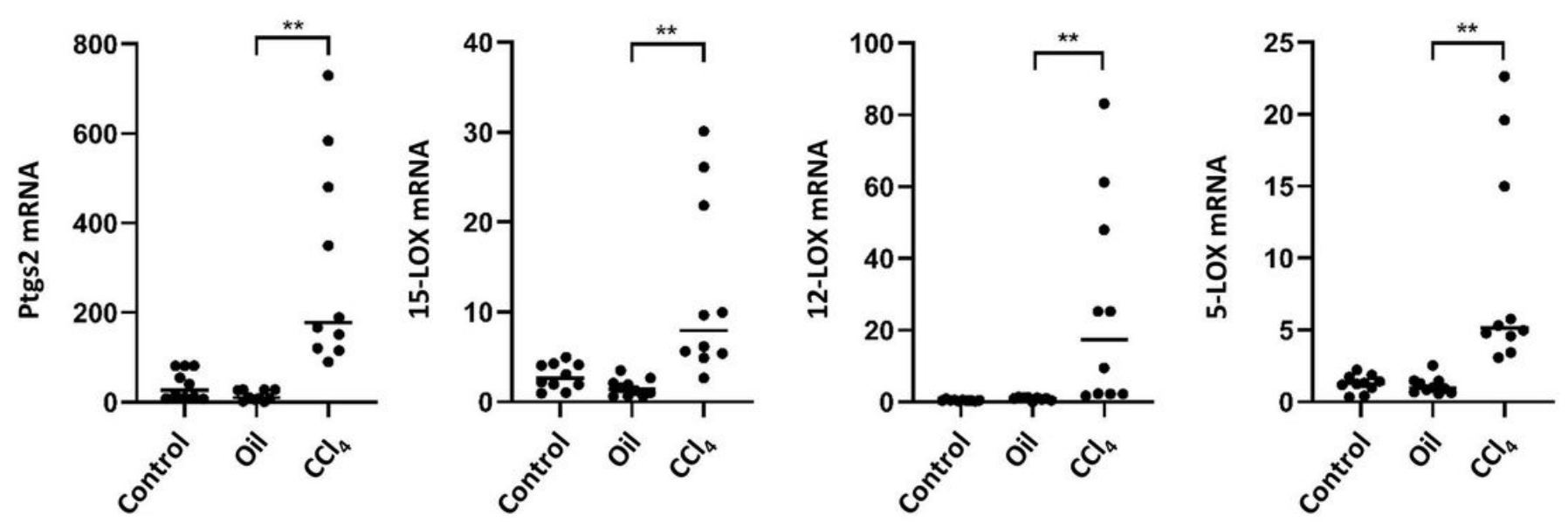

C
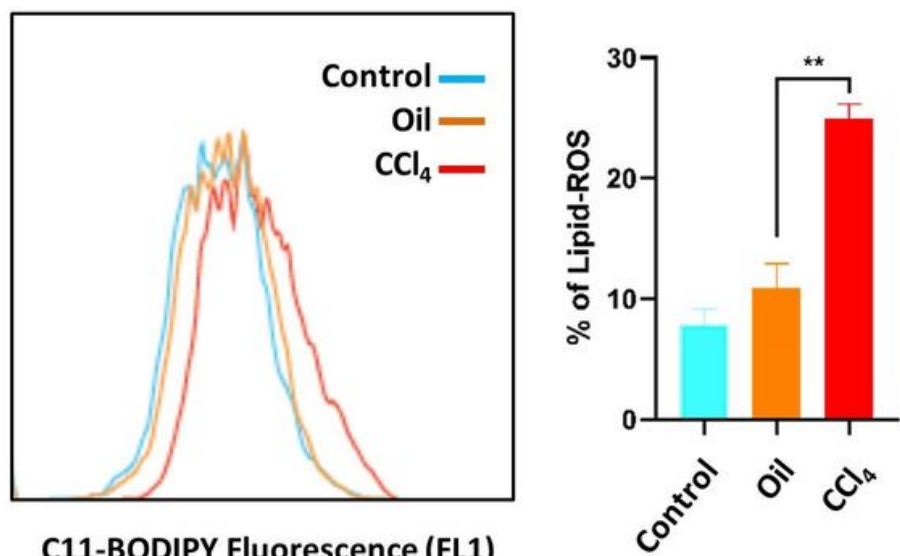

D

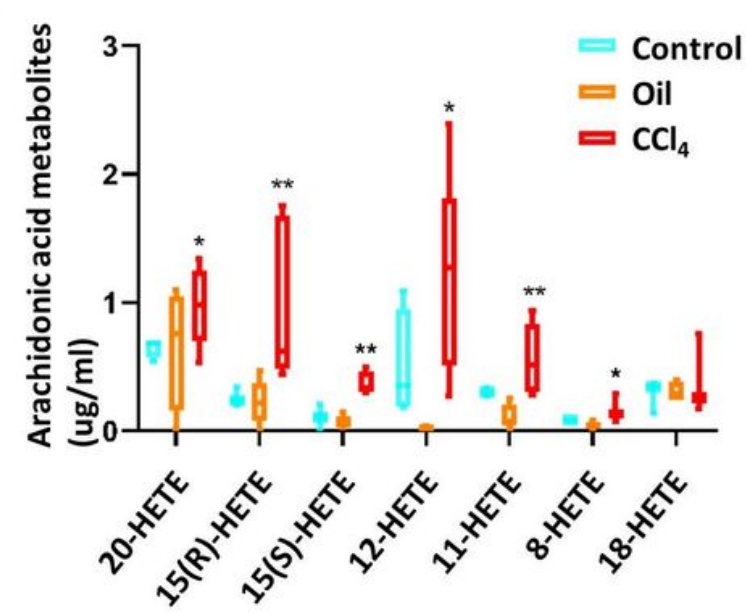

Figure 1

Ferroptosis occurs in the liver tissues of an ALI mouse model. (A) Hematoxylin and eosin (HE) staining shows that administration of CCl4 induces ALI in mice. (B) Ptgs2, 15-LOX, 12-LOX, and 5-LOX mRNA levels were measured in the livers of 8-week-old male C57BL/ 6 mice treated for $6 \mathrm{~h}$ with or without CCl4. The mRNA levels were normalized to that of GAPDH and are expressed relative to the mean value in the PBS-treated mice. (C) Lipid peroxidation was measured using C11-BODIPY staining. (D) UPLC-MS/MS analysis of of 20-hydroxyeicosatetraenoic acid (HETE), 15(R)-HETE, 15(S)-HETE, 12-HETE, 11-HETE, 8- 
HETE, and 18-HETE were performed in liver tissues. Significance was calculated using one-way ANOVA with Tukey's post-hoc test. ${ }^{*} p<0.05$ or ${ }^{* \star} p<0.001$ indicates a significant difference between groups.

A
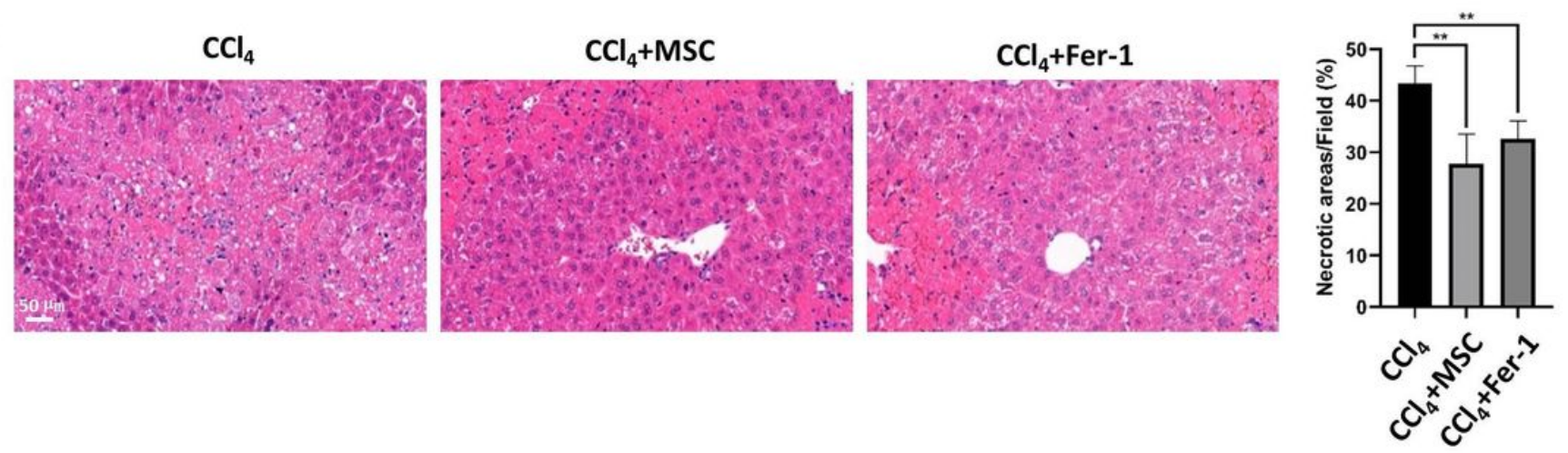

B
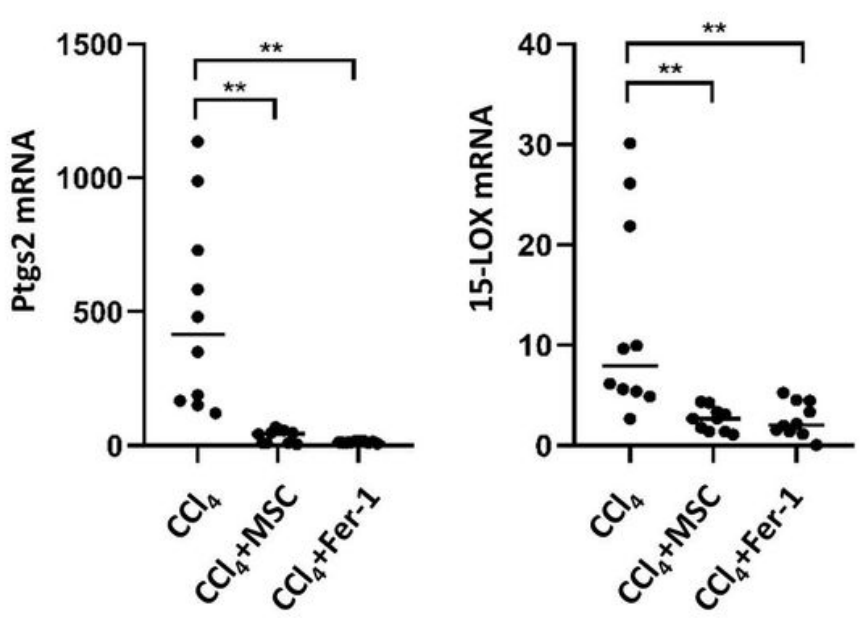

C

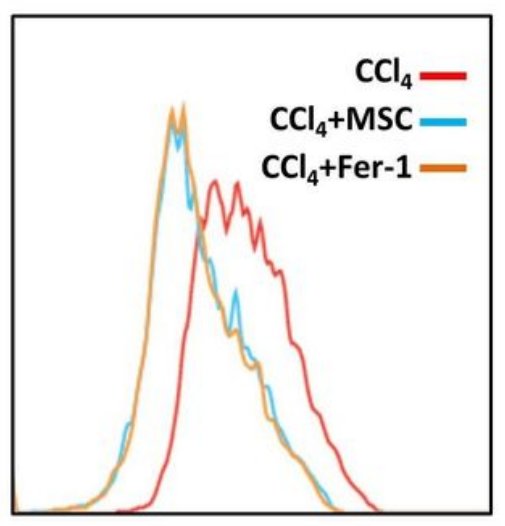

C11-BODIPY Fluorescence (FL1)

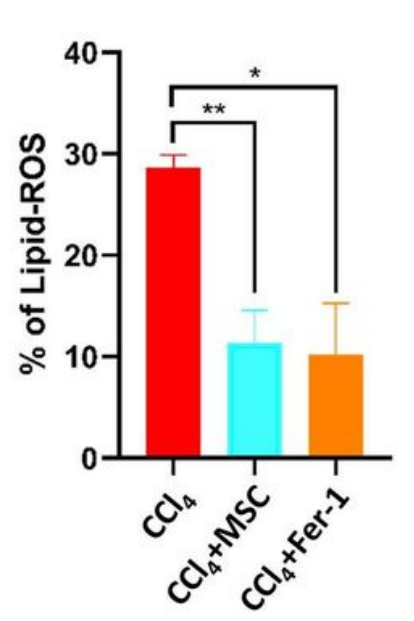

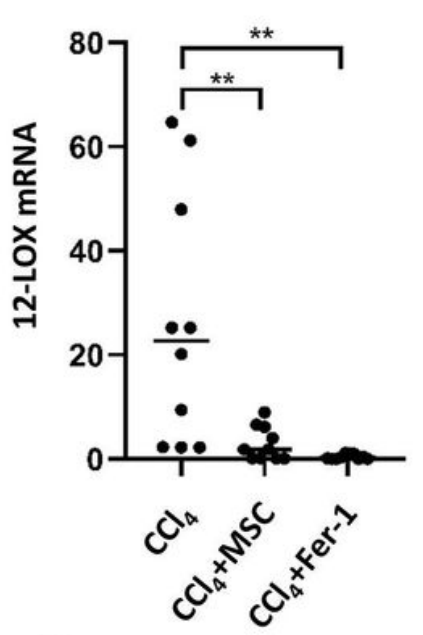

D

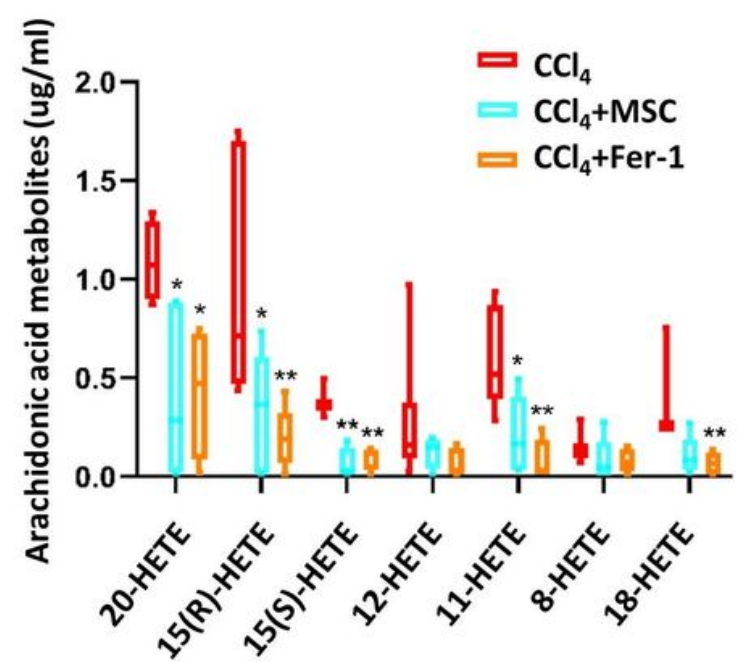

Figure 2

MSC transplantation prevents CCl4-induced ferroptosis in ALI. (A) HE staining shows that MSC transplantation and treatment with ferrostatin-1 (Fer-1), but not erastin, alleviated CCl4-induced ALI. (B) Ptgs2, 15-LOX, 12-LOX, and 5-LOX mRNA levels were measured in mouse livers of the CCI4, MSC, erastin, and Fer-1 groups. The mRNA levels in panel B were normalized to that of GAPDH. (C) Lipid peroxidation was measured by C11-BODIPY staining. Lipid peroxidation of hepatocytes in the liver was significantly 
increased in the $\mathrm{CCl} 4$ and erastin groups but significantly reduced in the MSC and Fer-1 groups. (D) UPLCMS/MS detection showed increased levels of 20-hydroxyeicosatetraenoic acid (HETE), 15(R)-HETE, 15(S)-HETE, 12-HETE, 11-HETE, 8-HETE, and 18-HETE in mouse livers of CCl4 group, and these effects were abrogated by MSC and Fer- 1 treatment. Significance was calculated by one-way ANOVA with Tukey's post-hoc test. ${ }^{*} p<0.05$ or ${ }^{* *} p<0.001$ indicates a significant difference between groups.

A
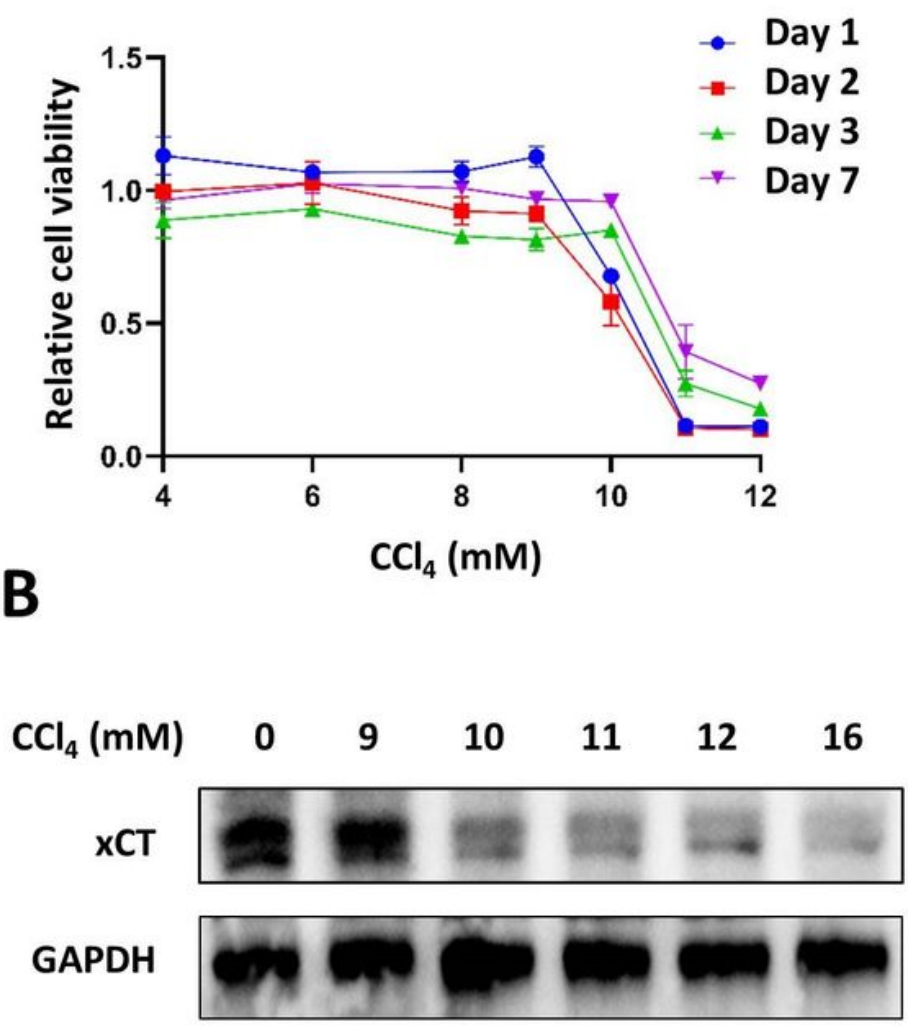

C

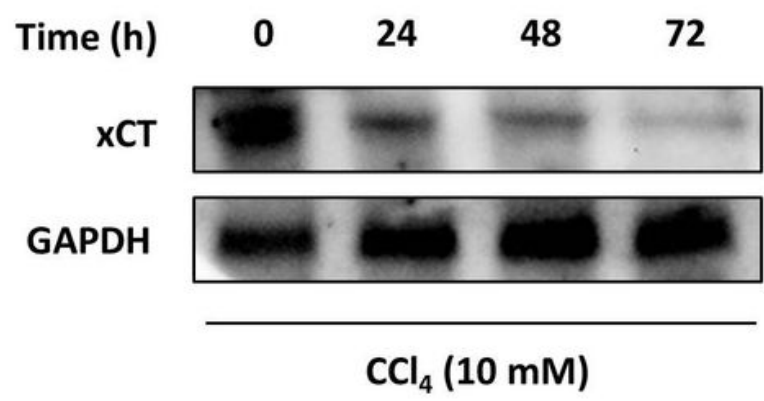

D
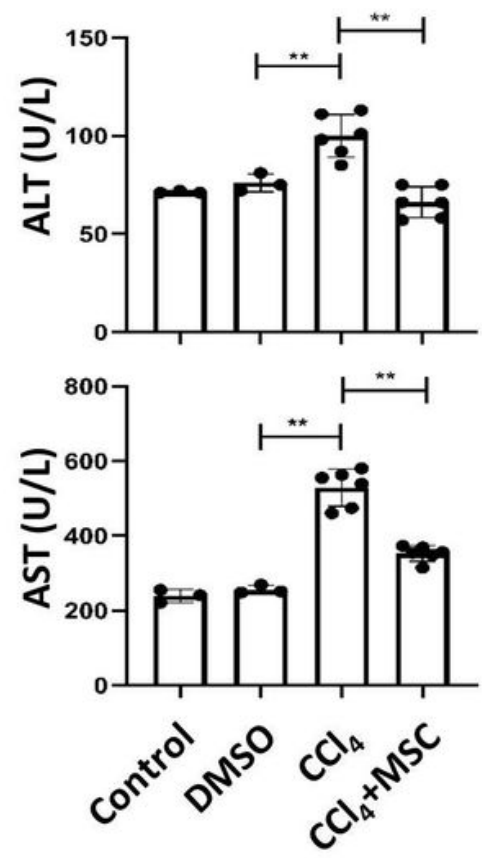

E

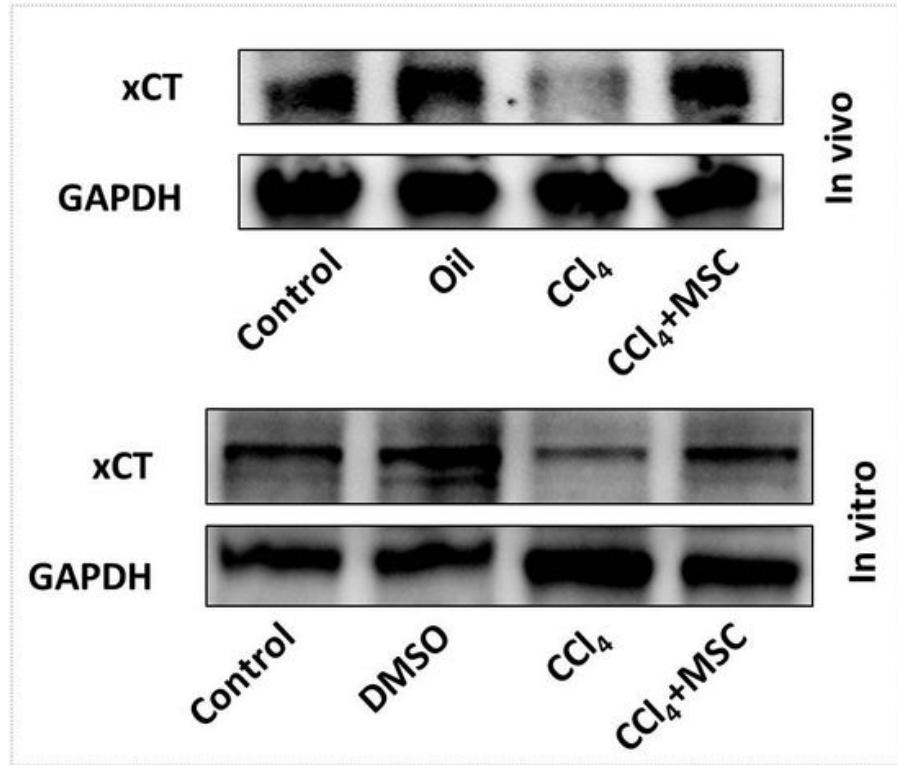

Figure 3 
The protein level of $\mathrm{XCT}$ is downregulated in CCl4-induced ALI and upregulated following MSC treatment in vivo and in vitro. (A) Cell viability was measured on days 1, 2, 3, and 7 after hepatocytes were treated with $4,6,8,9,10,11$, and $12 \mathrm{mM} \mathrm{CCl} 4$. (B) Western blot analysis was performed to measure the $\mathrm{xCT}$ protein level in mouse primary hepatocytes treated with $0,9,10,11,12$, and $16 \mathrm{mM} \mathrm{CCl} 4$ for $24 \mathrm{~h}$. (C) or with $10 \mathrm{mM} \mathrm{CCl} 4$ for $0,24,48$, and $72 \mathrm{~h}$. (D) Co-culture of primary hepatocytes with MSC significantly increased the levels of AST and ALT induced by CCI4. (E) MSC treatment significantly reduced the downregulated $\mathrm{xCT}$ protein levels induced by $\mathrm{CCl} 4$ in vivo and in vitro. The mRNA levels in panel $\mathrm{F}$ were normalized to that of GAPDH. Significance was calculated by one-way ANOVA with Tukey's post-hoc test. ${ }^{*} \mathrm{p}<0.05$ or ${ }^{* *} \mathrm{p}<0.001$ indicates a significant difference between groups.

A
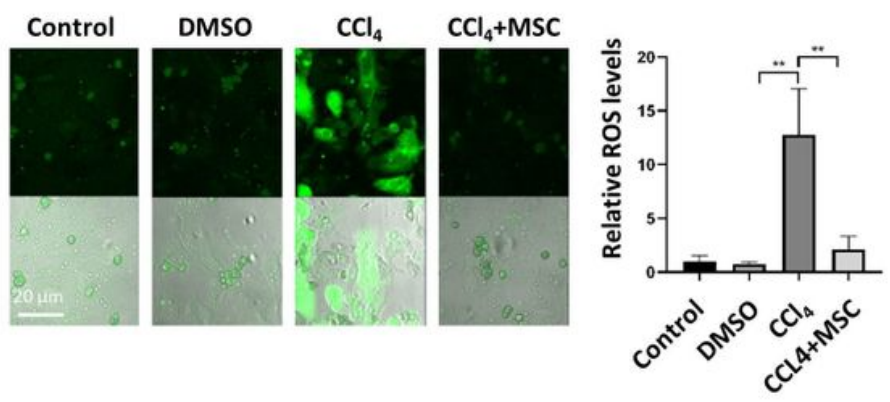

B

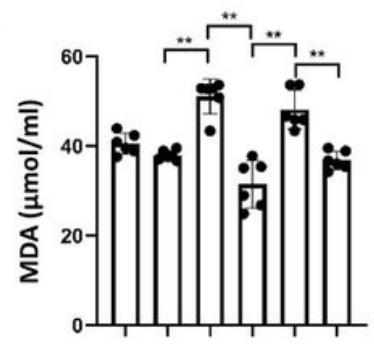

D
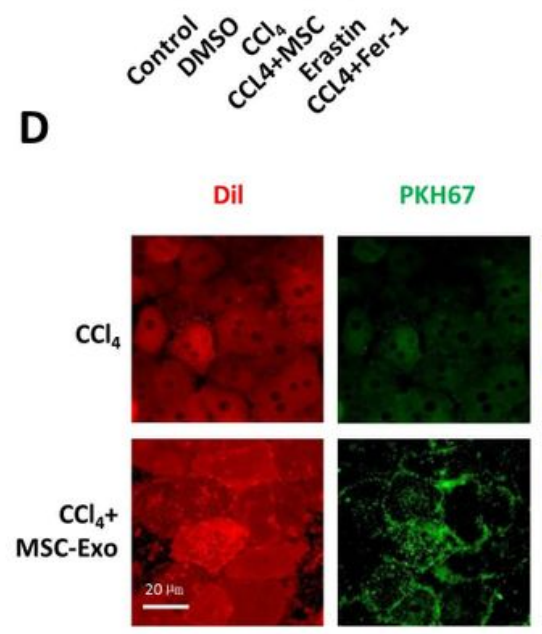

C

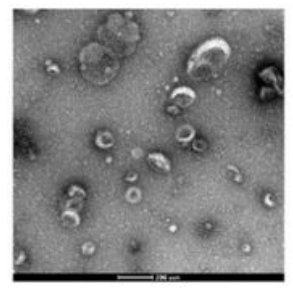

Exosomes

DAPI

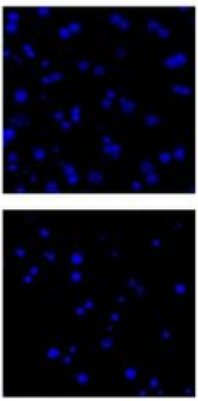

$\mathbf{E}$

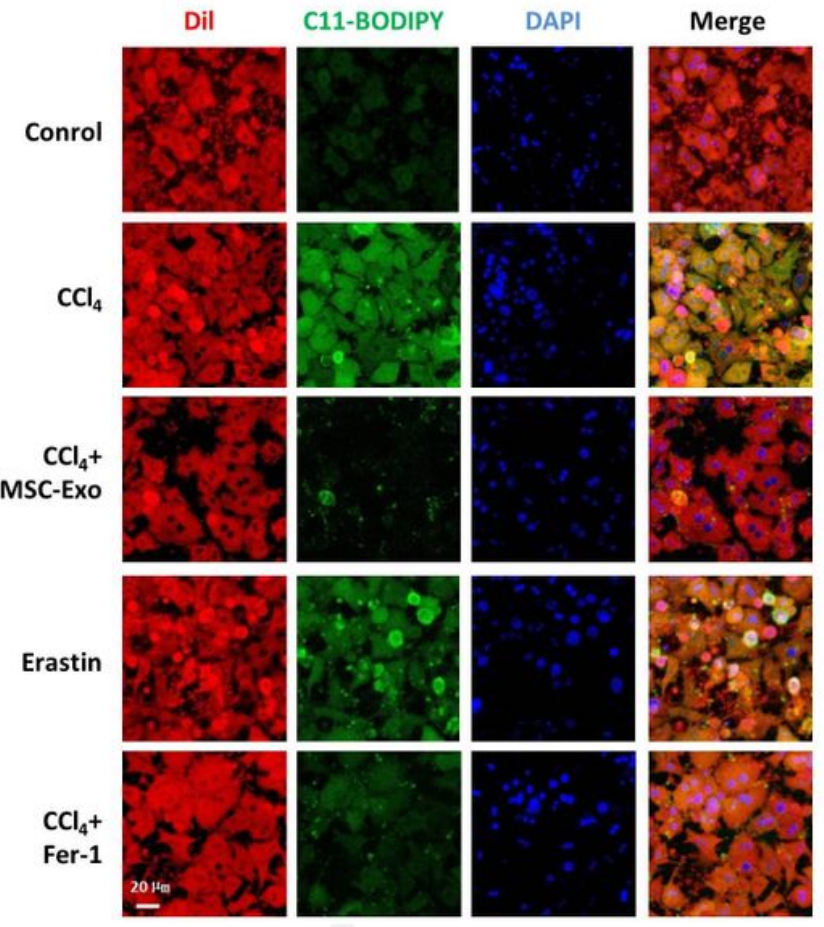

$\mathbf{F}$

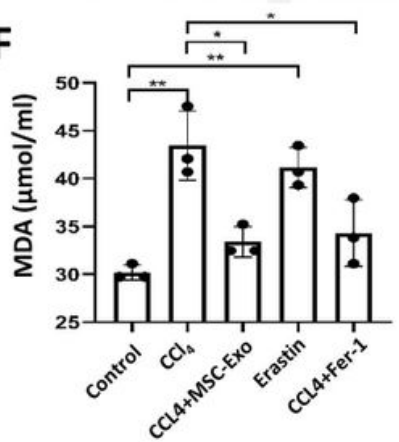

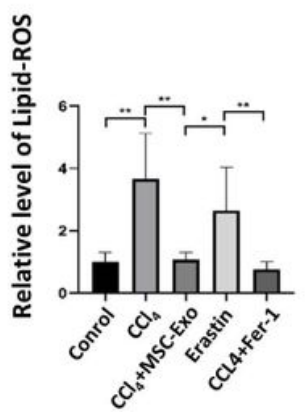

Figure 4

Exosomes mediate the effects of MSCs against CCl4-induced ferroptosis. (A) Relative ROS levels were measured by H2DCFDA staining in primary hepatocytes treated with PBS, DMSO, or $10 \mathrm{mM} \mathrm{CCl} 4$ for $24 \mathrm{~h}$ with or without MSC co-culture. (B) The increased MDA level induced by $\mathrm{CCl} 4$ was downregulated by MSC co-culture and Fer-1 treatment in primary hepatocytes. (C) Refrigerated transmission electron microscopy 
was used to assess exosomes isolated from MSCs. (D) PKH67 lipid dye was aggregated on cell membranes treated with PKH67-labeled exosomes in the CCl4 group. Exosomes were derived from MSCs and incubated with normal primary hepatocytes or $\mathrm{CCl} 4$-induced acute injured hepatocytes for $24 \mathrm{~h}$. (E) C11-BODIPY staining showed a significant increase in lipid peroxidation in hepatocytes following $\mathrm{CCl} 4$ and erastin treatment, while MSC-Exo and Fer-1 downregulated the increased lipid peroxidation induced by $\mathrm{CCl} 4$. (F) The increased MDA level induced by $\mathrm{CCl} 4$ was downregulated by MSC-Exo and Fer-1 treatments in primary hepatocytes. Significance was calculated by one-way ANOVA with Tukey's post-hoc test. ${ }^{*} p<0.05$ or ${ }^{* \star} p<0.001$ indicates a significant difference between groups.

A
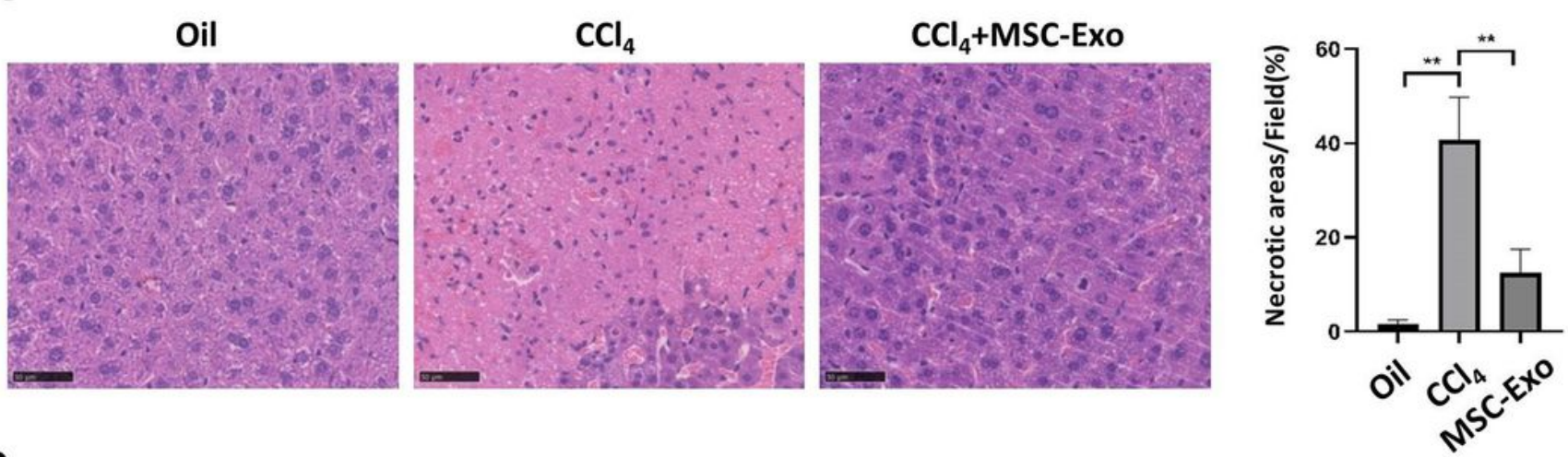

B
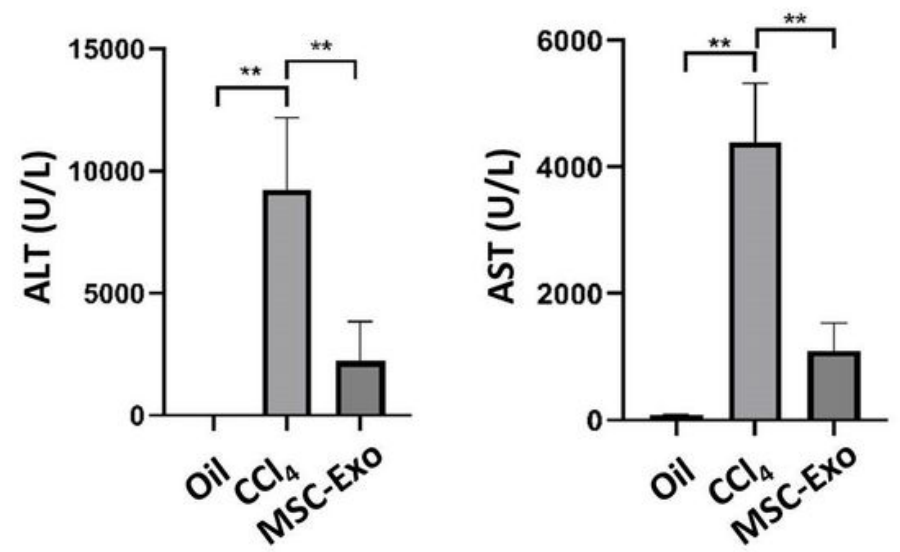

C

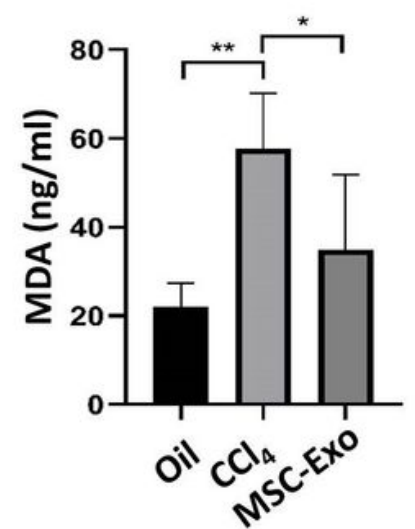

D
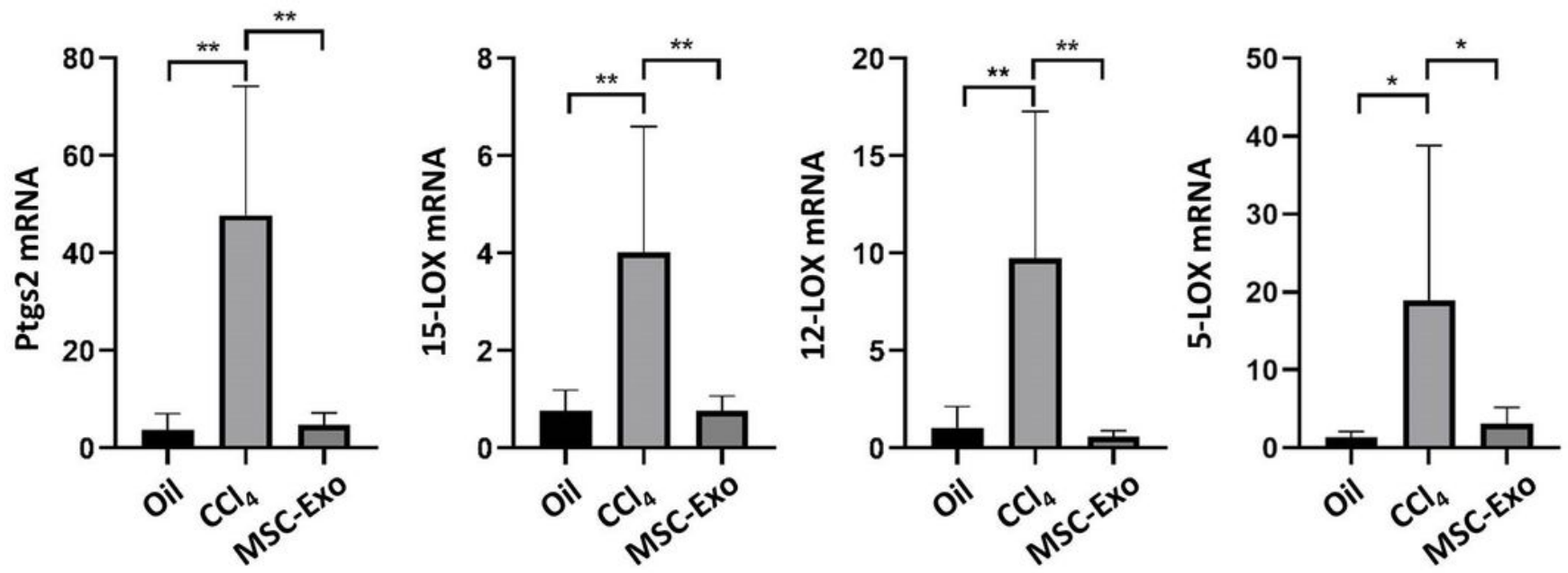


\section{Figure 5}

Exosomes mediated the effects of MSCs against CCl4-induced ferroptosis. (A) HE staining shows that MSC-Exo treatment alleviated CCl4-induced ALI. (B) MSC-Exo dramatically reversed the increased serum levels of ALT and AST induced by CCI4. (C) UPLC-MS/MS detection indicated significant upregulation of the serum MDA level induced by $\mathrm{CCl} 4$, which was downregulated following MSC-Exo treatment. (D) MSCExo treatment significantly reversed the increased mRNA levels of Ptgs2, 15-LOX, 12-LOX, and 5-LOX induced by $\mathrm{CCl} 4$ in the liver.
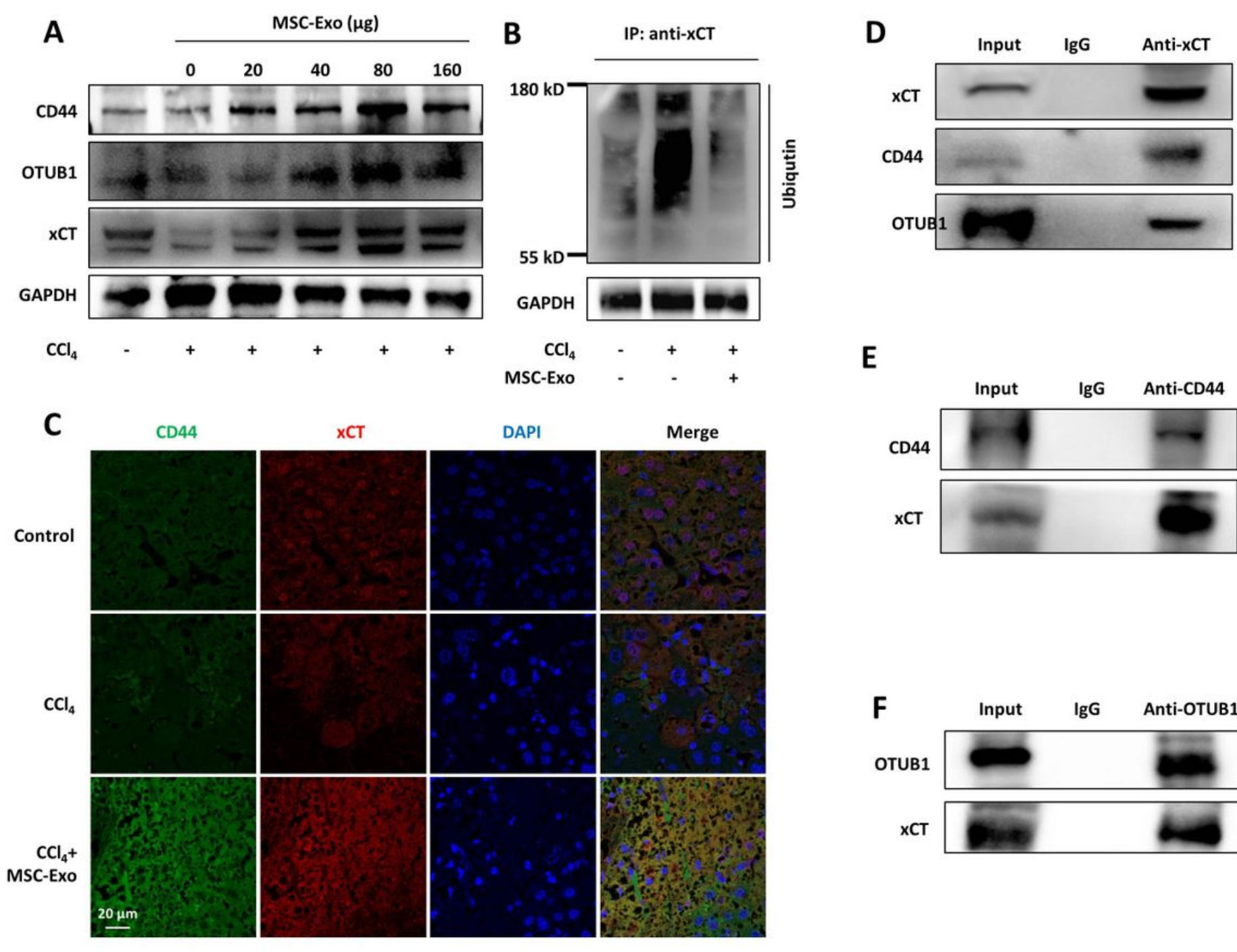

E
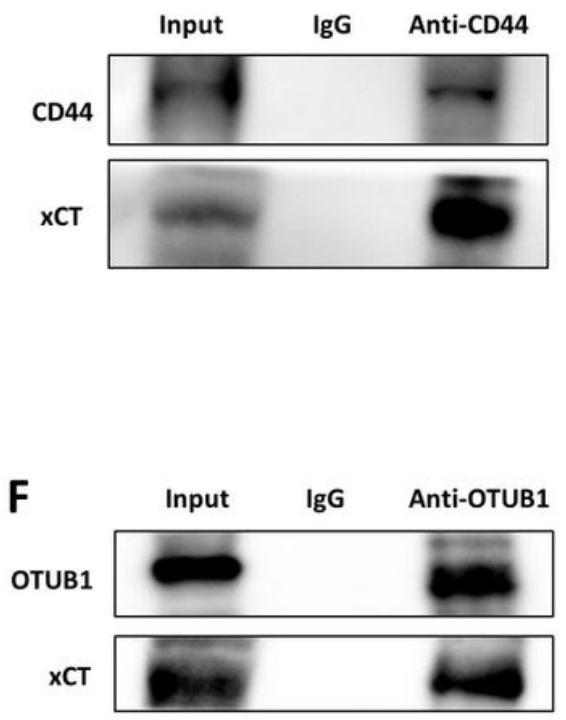

\section{Figure 6}

MSC-Exo protect against ferroptosis via the CD44-xCT axis in ALI. (A) CD44, OTUB1 and xCT protein levels in CCl4-induced acute injured hepatocytes treated with 0, 20, 40, 80, and $160 \mu \mathrm{g} \mathrm{MSC-Exo} \mathrm{were}$ detected by Western blot analysis. (B) CCl4-induced ALI increased ubiquitination of XCT in hepatocytes, and this effect was downregulated following MSC-Exo treatment. (C) Immunofluorescence assays were used to detect the protein levels of CD44 and XCT in liver tissues. (D) Western blot analysis of CD44 and 
OTUB1 after co-immunoprecipitation of anti-xCT from liver tissue in the CCl4 group treated with MSC-Exo. One percent of the sample was loaded as input. (E) Western blot analysis of XCT after coimmunoprecipitation of anti-CD44 from liver tissue in the $\mathrm{CCl} 4$ group treated with MSC-Exo. One percent of the sample was loaded as input. (F) Western blot analysis of XCT after co-immunoprecipitation of antiOTUB1 from liver tissue in the CCl4 group treated with MSC-Exo. One percent of the sample was loaded as input. The mRNA levels in panel D were normalized to that of GAPDH. Significance was calculated by one-way ANOVA with Tukey's post-hoc test. ${ }^{*} p<0.05$ or ${ }^{* *} p<0.001$ indicates a significant difference between groups. 
A

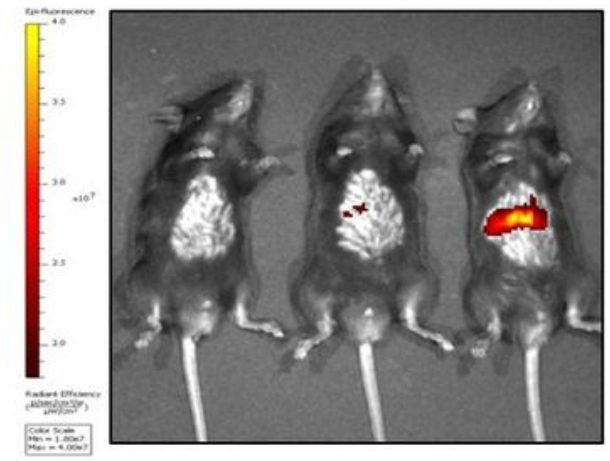

B
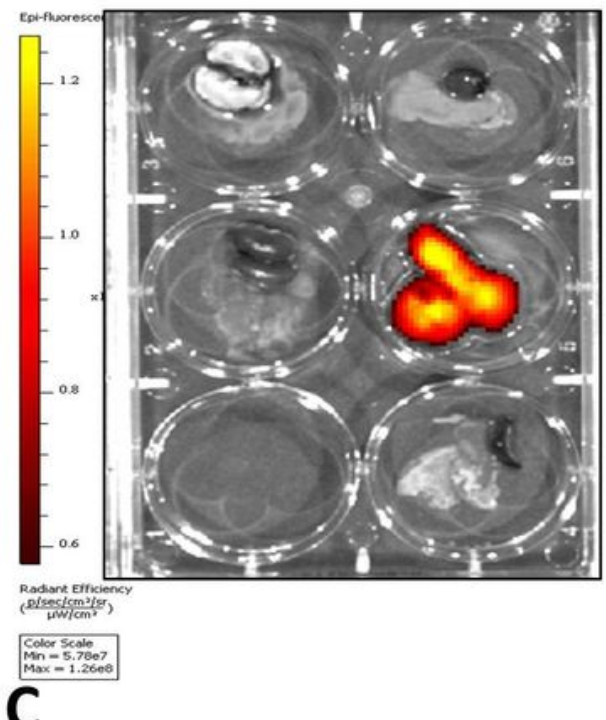

C

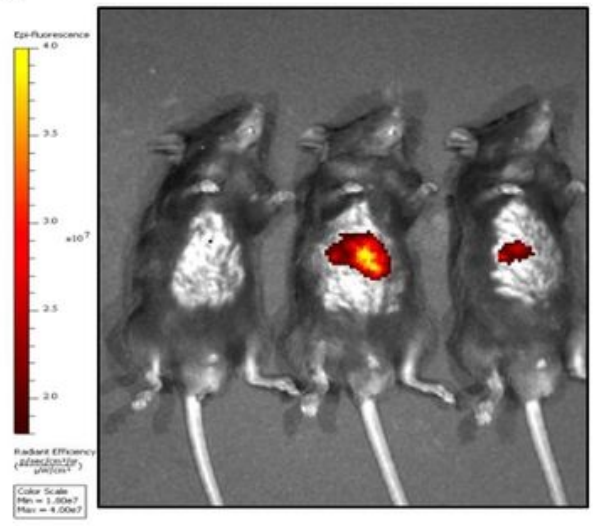

MSC-Exo (CD44 Blocked)
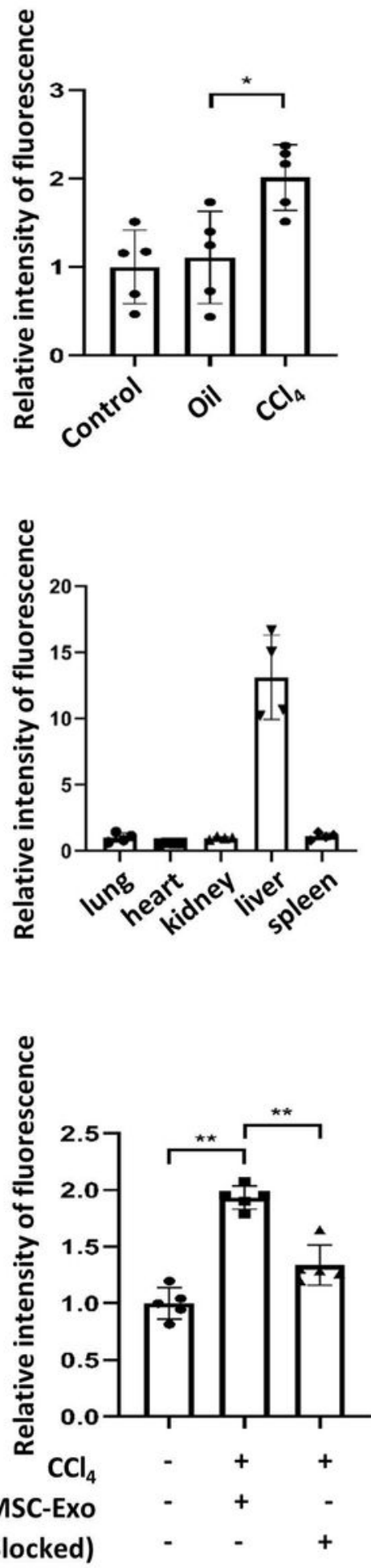

Figure 7

Exosomes in the circulation were more readily localized in the liver in ALI mice. (A) Whole-body fluorescence imaging of C57BL/ 6 male mice treated with $8 \mathrm{mg} / \mathrm{kg}$ labeled exosomes from MSC conditioned medium. Images were taken $6 \mathrm{~h}$ after tail vein injection. (B) Quantitative fluorescent imaging of lung, heart, kidney, liver, and spleen from recipient mice receiving labeled exosomes from MSC conditioned medium. (C) Neutralizing antibodies against CD44 reduced hepatic engraftment of MSC-Exo 
in ALI murine livers. Images were taken at $4 \mathrm{~h}$. Data are the mean $\pm \mathrm{SD}$ fluorescence; ${ }^{*} \mathrm{p}<0.05,{ }^{*} \mathrm{p}<$ $0.001 ; n=5$. Individual images are presented. (D) Schematic diagram of the protective effects of MSCderived exosomes on maintaining xCT function during ferroptosis involved in CCl4-induced ALI.

\section{Supplementary Files}

This is a list of supplementary files associated with this preprint. Click to download.

- GraphicalAbstract.jpg

- supplementarymaterials.docx 